\title{
LADA (latent autoimmune diabetes in adults) in Norway - occurrence, risk factors, treatment and complications
}

\author{
Sofia Carlsson ${ }^{1}$, Kristian Midthjell ${ }^{2}$ and Valdemar Grill ${ }^{3}$ \\ 1) Department of Epidemiology, Institute of Environmental Medicine, Karolinska Institutet, Stockholm, Sweden \\ 2) HUNT Research Centre, Department of Community Medicine and General Practice, Norwegian University of \\ Science and Technology, Levanger, Norway \\ 3) Department of Cancer Research and Molecular Medicine, Norwegian University of Science and Technology, \\ and Trondheim University Hospital, Trondheim, Norway \\ Correspondence: Sofia Carlsson, Institute of Environmental Medicine, Karolinska Institutet, S-171 77 Stockholm, Sweden \\ E-mail: sofia.carlsson@ki.se Telephone: +46852480011Ｔelefax: +46831 3961
}

\begin{abstract}
LADA (latent autoimmune diabetes in adults) may be the most common form of autoimmune diabetes and thus encompasses a significant proportion of the diabetic population. Still, knowledge about this common form of diabetes is limited. In this paper we give an overview of some aspects of LADA, primarily using data from the Nord-Trøndelag Health Study (HUNT). In HUNT, LADA accounts for 7\% of all incident cases of diabetes in adults. Data from HUNT indicate that risk factors for LADA include overweight, physical inactivity, family history of diabetes and low psychosocial well-being. Risk factors thus seem to be largely similar to those of type 2 diabetes which suggests a partially shared pathogenesis, in spite of the autoimmune nature of LADA. With regard to genetic factors LADA may be a genetic mix of type 1 and type 2 diabetes, as LADA patients seem to have an increased prevalence of HLA haplotypes linked to type 1 diabetes, and possibly also genes associated with type 2 diabetes. Patients with LADA are at increased mortality risk, primarily from cardiovascular disease. The excess risk seems to be linked to poor glycemic control rather than metabolic risk factors. These results emphasise the importance of optimal treatment modalities to improve survival in LADA. Documentation of optimal treatment for LADA is still lacking and there is an urgent need for studies in this field.
\end{abstract}

\section{INTRODUCTION}

Like type 1 diabetes in children, LADA is an autoimmune form of diabetes characterized by antibodies against the insulin-producing beta cells in the pancreas $[1,2]$. The disease develops in adulthood and compared to traditional type 1 diabetes, it progresses more slowly and requires insulin treatment at a later stage $[1,2]$. LADA also seems to share some features with type 2 diabetes since LADA patients tend to be insulin resistant [3]. Epidemiological studies indicate that LADA may account for $2-12 \%$ of all cases of diabetes [4]. In line with this, findings of the Nord-Trøndelag Health Study (HUNT) show that LADA accounts for $7 \%$ of all new cases of diabetes in adults. LADA thus encompasses a significant proportion of the diabetic population. Knowledge about LADA is still limited, including its etiology, treatment and long term consequences. In this paper we will give an overview of some aspects of LADA, primarily using data from the HUNT study.

The criteria for LADA are debated. Age, antibody positivity and independence of insulin treatment have been used to diagnose LADA. Each of these criteria has been challenged. The age limit (usually $<30$ years) is to some extent arbitrary. Antibody positivity is regarded as a sine qua non for diagnosis of LADA. The presence of positivity (usually anti-GAD-antibodies against glutamic acid decarboxylase) in the non-diabetic popu- lation has been used as argument of non-specificity, especially in cases of diabetes who display low titres of anti-GAD [5]. However, recent data from the HUNT surveys demonstrate that anti-GAD positivity in long term ( $>10$ years) non-diabetic individuals associate to other markers of autoimmunity [6]. Hence, the antiGAD findings in non-diabetic individuals cannot easily be dismissed as false positives.

Is it possible to have LADA without being antibody positive? Data, again from the HUNT surveys do indicate so. Of the individuals with diabetes who were found to have LADA at HUNT2 because they were anti-GAD positive, about half had lost anti-GAD positivity when they were re-examined at HUNT3 [7]. Hence, if diabetes had been diagnosed for the first time at HUNT3 these individuals would have been classified as having type 2 diabetes. If one accepts that even fleeting positivity for autoimmune markers in diabetes does have clinical importance, then one must conclude that "mild" autoimmunity plays a role for developing diabetes not only for those individuals that are diagnosed as LADA at a given time point of examination but also for a significant part of those individuals who are currently diagnosed as having type 2 diabetes.

The third criterion that has been used to diagnose LADA is independence of insulin treatment at diagnosis and for a significant period of time thereafter. Whether an individual with new-onset diabetes is in need of insulin treatment is open to subjective evalua- 


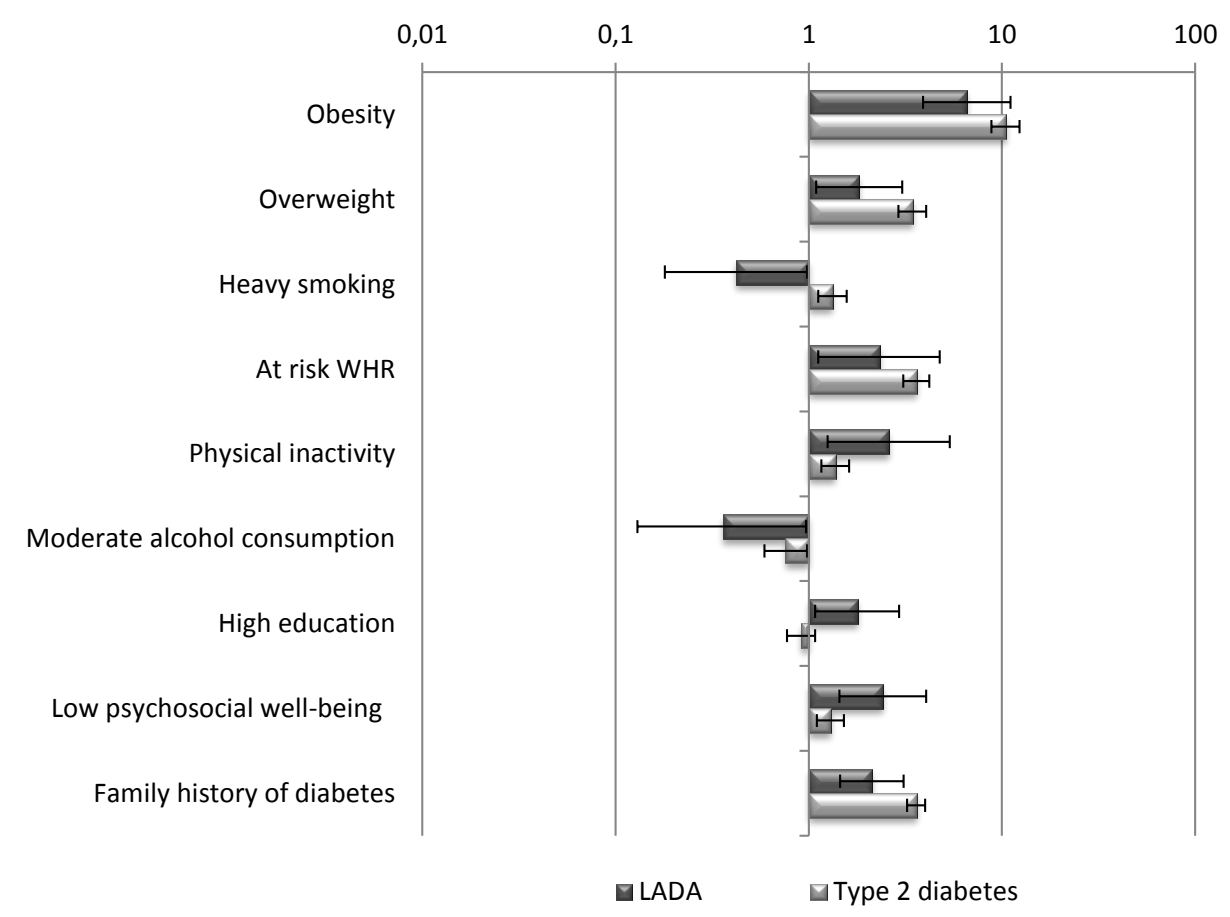

Figure 1. Hazard ratios of LADA and type 2 diabetes in relation to potential risk factors, results from the HUNT Study 1984-2008.

tion and to current norms of treatment, which may differ between countries and regions. Also whether individuals are insulin resistant or not will influence any need for exogenous insulin. Clearly, the insulin independence criterion is the fussiest one when defining LADA. Accordingly, it has been proposed to merge LADA into a single category of autoimmune diabetes in adults [8]. Several papers, including some of our own, have recently used the latter categorisation. Still, the insulin independence feature separates LADA patients phenotypically and genetically from those with adult onset "classical" type 1 diabetes [9].

\section{RISK FACTORS}

Since LADA has features of both type 1 and type 2 diabetes it seems possible that LADA is caused by: 1) the same underlying disease processes as traditional type 1 diabetes. But the disease may also have a 2) partially unique etiology, or 3) an etiology that includes risk factors for both type 1 and type 2 diabetes. To date, few attempts have been made to clarify this. The lack of studies on LADA is probably attributable to the fact that in most observational studies on diabetes, the necessary data (indicators of autoimmunity) to separate LADA from type 2 diabetes have not been collected. One exception is the Norwegian HUNT Study where the incidence of LADA can be followed 1984-2008. With these data at hand we have studied some potential risk factors of LADA, including genetic factors [9], family history of diabetes, overweight, physical activity, smoking, alcohol consumption, psychosocial and socioeconomic factors (Figure 1) [10-15].

\section{HUNT STUDY}

HUNT is based on three extensive cross-sectional health investigations conducted in Nord-Trøndelag in 1984-86, 1995-97 and 2006-08 [16]. On these occasions, all adult inhabitants ( $\geq 20$ years old) in the county were invited to a clinical investigation which included extensive questionnaires with items on health and lifestyle factors. Based on these cross-sectional investigations, a cohort of about 117000 people, who participated in at least two HUNT surveys, could be formed and followed 11-22 years with regard to incidence of diabetes (Figure 2). To study mortality, we have formed a cohort of $\sim 65000$ subjects (3100 prevalent cases of diabetes) based on HUNT2, that can be followed from 1995 to 2009 through the Norwegian cause of death registry. Patients were classified according to diabetes type based on measures of anti-GAD together with age at onset: Our definition of LADA was age $\geq 35$ years at diagnosis and anti-GAD positive $(\geq 0.08)$. Since there is at present no established definition of LADA, we also used an alternative, more stringent definition including the additional criterion that the patient should not have been treated with insulin during the first year after diagnosis [4]. With this second definition, we get similar results as those presented here, but fewer cases (since information on treatment was only available for $83 \%$ of cases) and therefore less precision.

\section{GENETIC FACTORS}

The genetic background of LADA has been investigated in relation to genes associated with type 1 as well as type 2 diabetes. A coupling to HLA risk haplotypes 


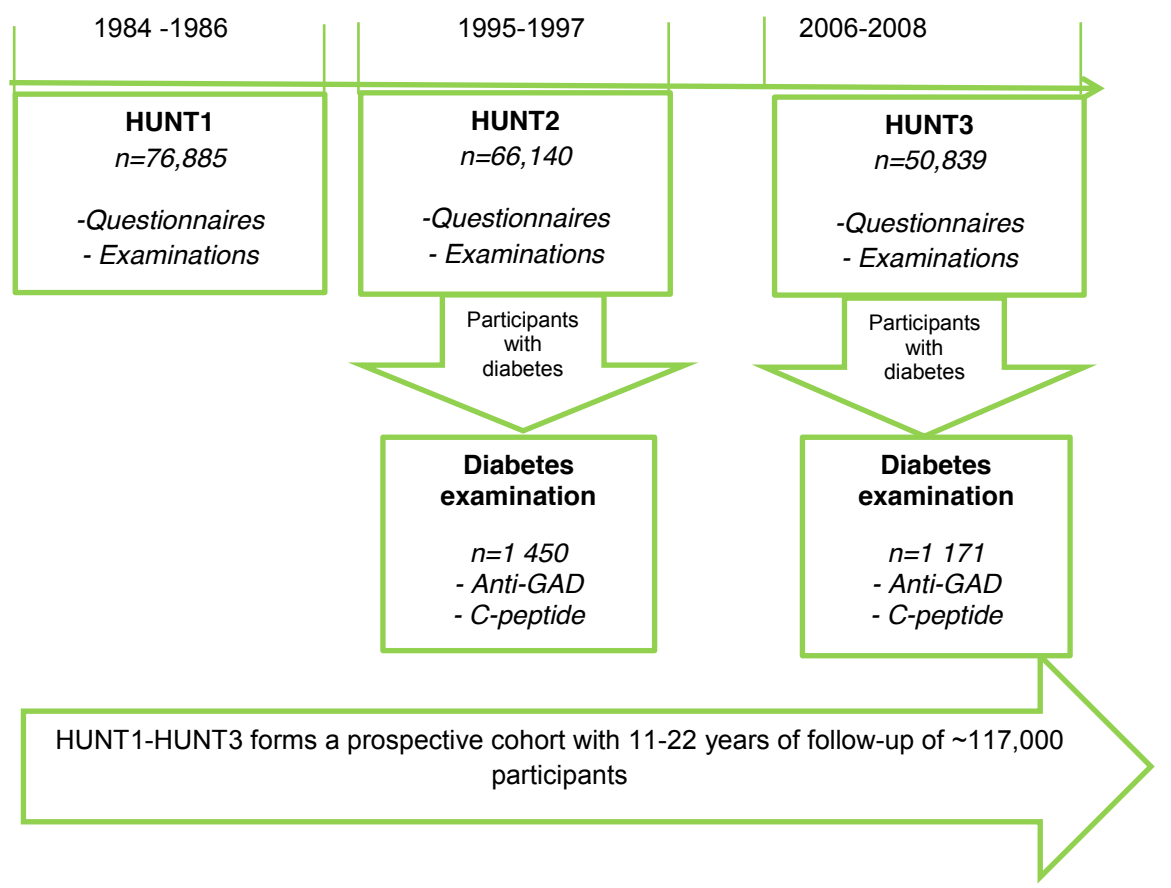

Figure 2. Study design, HUNT.

has been documented $[9,17]$. Interestingly, the strength of this association correlates positively with titers of anti-GAD [9]; however, a complete correspondence is not seen. Whether a cause-effect relationship exists between the risk haplotypes and the presence and strength of antibodies is still unknown. Also the PTPN22 gene associates with LADA whereas a relationship with the INS gene is disputed and was not found in the HUNT study, but was, as expected, found in subjects with "classical" type 1 diabetes [9]. As to genes associated with risk for type 2 diabetes, one study found an association with TCFL7 [18], i.e. the gene which is to date most strongly associated with type 2 diabetes; however in the HUNT study this finding could not be reproduced [9] whereas an association was found with the FTO gene (which is associated both with overweight and type 2 diabetes).

To sum up, a relationship between type 1 diabetes associated genes and LADA seems well proven although the genetic background behind autoimmunity is not identical with that of type 1 diabetes. A coupling with type 2 diabetes genes is at present not completely documented. Uncertainties stem at least partly from the heterogeneity of the LADA population.

\section{ENVIRONMENTAL FACTORS}

Results from HUNT suggest that traditional risk factors such as family history of diabetes and obesity are as strong risk factors for LADA as for type 2 diabetes $[10,11]$. Recent findings also suggest an increased risk linked to poor psychosocial well-being and sleeping problems [12]. On the other hand, a protective effect seems to be conferred by physical activity and, also in correspondence with type 2 diabetes, by moderate alcohol consumption [15]. Risk factors of LADA thus include factors known to affect insulin sensitivity [1921]. These findings fit with other data indicating that LADA is characterized by insulin resistance [3]. LADA thus shares features of type 2 diabetes despite its coupling to type 1-like autoimmunity. This highlights the interactions that are bound to take place between insulin resistance and faulty insulin secretion (for LADA due to autoimmunity) in the process of developing diabetes.

There are however discrepancies; smoking is associated with a reduced risk of LADA [14], possibly through an inhibitory effect of nicotine on autoimmunity. The risk of LADA is also twice as high for people with high socioeconomic status [13], while type 2 diabetes, in this and many previous studies [22] is more common for people with low socioeconomic status. Similar results have been reported previously for classical type 1 diabetes, which was more prevalent in children of high-income families [23]. The increased risk which we observed was not explained by traditional risk factors such as family history, obesity and smoking, but may result from other environmental factors that differ between socioeconomic groups. High education was also associated with higher levels of anti-GAD. This suggests that environmental factors linked to development of autoimmunity may explain the excess risk associated with high education. Proposed explanations for this finding includes "the hygiene hypothesis" which assigns importance to a lower prevalence of infections early in life [24], or differences in dietary pattern habits, that may affect an autoimmune process. Unfortunately, there are limited data on early infections and diet in HUNT and additional studies are therefore needed to further test the hypothesis. 


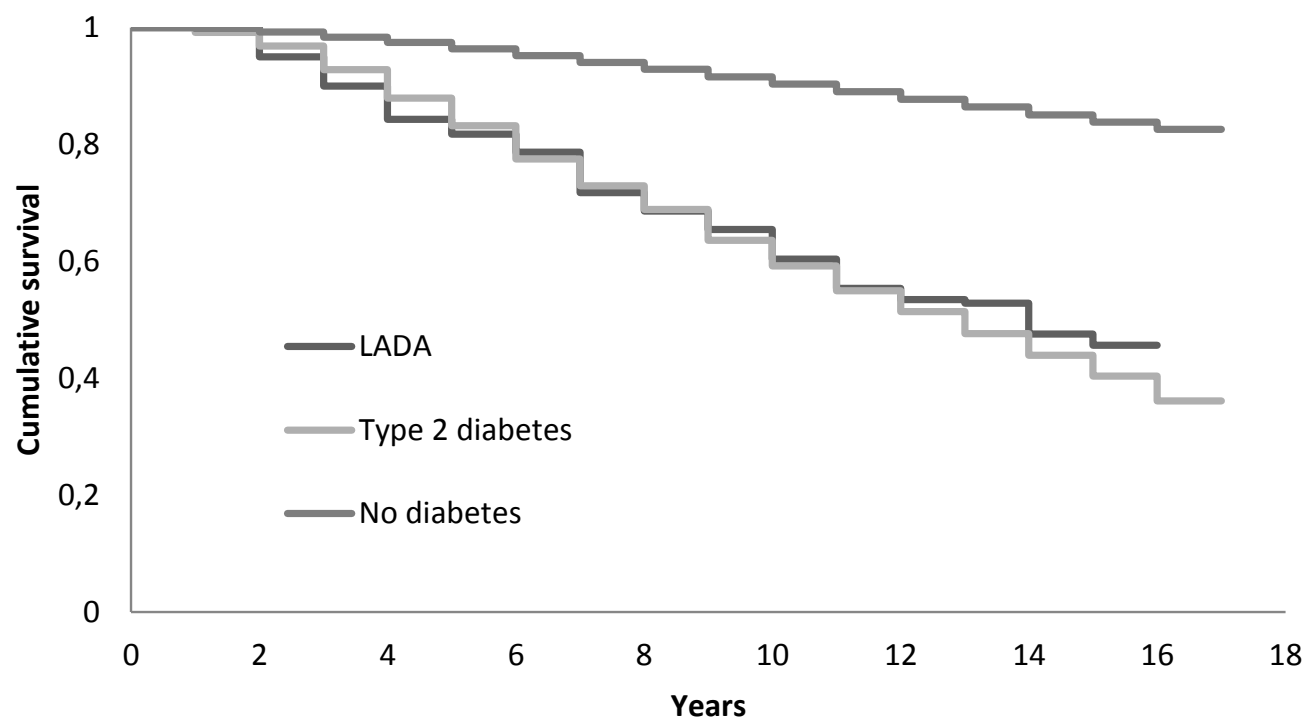

Figure 3. Kaplan-Meyer curves of mortality in LADA and type 2 diabetes. Results from the HUNT Study 1995-2009.

\section{TreatMenT}

Is treatment of LADA patients different from type 2 diabetes? - or should it be? LADA was treated as type 2 diabetes before measurements of anti-GAD were possible in a clinical setting. After the advent of routine measurements several years elapsed before the concept of LADA was fully realized by most practicing physicians. In practical terms this has meant that few, if any, physicians in Nord-Trøndelag were aware of the potential differences in progression of disease vs. type 2 diabetes at the time of HUNT2. From a scientific point of view this has facilitated the characterization of "natural" progression of LADA in terms of need for insulin treatment as detailed in some of our studies [25]. Still, data from HUNT3 (unpublished) indicate that initial treatment of LADA continues in most cases to be similar to that of type 2 diabetes.

Data from a Japanese study indicate that early insulin treatment (diverting from the norm of treating type 2 diabetes) is beneficial in upholding endogenous insulin secretion [26], in turn being beneficial for metabolic control and avoidance of diabetic complications [27]. However, the utility of early insulin treatment has been debated. A recent Cochrane review has concluded that new and unbiased studies are needed to test the utility of early insulin treatment [28]. To this end, one of us (VG) has initiated a Nordic study to test the concept. The BALAD study (Behandling Av LADA, Treatment of LADA) tests for effects of exogenous insulin as add-on to ongoing treatment with metformin. The rationale for co-treatment with metformin is based on the observation that most LADA patients, at least in the Nordic countries, are overweight and therefore insulin resistant. Therefore, it seems important that a clinical study incorporates treatment of insulin resistance. The use of metformin serves this purpose. The study is on-going and patients are still being recruited.

\section{Complications}

The long term consequences of LADA in terms of mortality and morbidity are largely unknown. Crosssectional studies indicate that these patients display features of the metabolic syndrome to a lower extent than patients with type 2 diabetes, but more than those with traditional type 1 diabetes [29-31]. In addition, these studies suggest that LADA patients tend to have worse glycemic control than patients with type 2 diabetes. One explanation could be that there is still no established treatment strategy for LADA [28], something which could make these patients more exposed to hyperglycemia and consequently, put them at increased risk of subsequent complications and mortality [32]. One could also speculate that because endogenous insulin production is lower, greater meal and exercise induced variations in blood glucose can be expected. Two studies based on British and Finnish populations have addressed mortality in autoimmune diabetes with adult onset/LADA $[33,34]$. These studies demonstrate similar [34] or even a tendency for higher mortality rates in LADA compared to type 2 diabetes [33]. Using data from HUNT and the national cause of death registry, we are currently studying survival in patients with LADA and type 2 diabetes between 1995 and 2009 [35]. Our findings indicate that subjects with LADA have a more favorable metabolic risk profile than subjects with type 2 diabetes including a lower prevalence of the metabolic syndrome (55\% vs. $77 \%)$ [35]. In spite of this, mortality in LADA patients was as high as in type 2 diabetes, including a $50 \%$ increased risk of all-cause mortality and a more than twofold increased risk of death from ishemic heart disease, compared to subjects without diabetes. The excess risk seemed mainly to be attributed to poor glycemic control, rather than metabolic risk factors or lifestyle. These results emphasise the importance of optimal 
blood glucose lowering treatment to improve survival of LADA patients.

\section{CONCLUSION}

LADA is a common form of diabetes with autoimmunity. However, risk factors seem partly similar to those of type 2 diabetes which suggests a partially shared pathogenesis with type 2 diabetes, possibly with insulin resistance as the common denominator. Accordingly LADA could be preventable or its onset delayed through modification of lifestyle, including normal weight, physical activity, moderate alcohol consumption and psychological well-being. However, it remains to be seen if the autoimmune component makes LADA less preventable through lifestyle changes than type 2 diabetes.

Patients with LADA are at increased mortality risk, primarily from cardiovascular disease. The excess risk seems to be linked to poor glycemic control. These results emphasise the importance of optimal treatment modalities to improve prognosis in LADA. General practitioners, who normally diagnose type 2 diabetes, should increase their attention to LADA, and measure anti-GAD and C-peptide in cases in where doubt exists concerning type 2 diabetes/LADA.

There is no established treatment strategy for LADA at present. Questions that remains to be addressed include whether early treatment with insulin could preserve insulin production in LADA patients. Treatment of LADA is an important area for future studies.

\section{ACKNOWLEDGEMENTS}

The authors wish to thank all the participants in the HUNTstudy and the staff carrying out the surveys. We also acknowledge the valuable contribution of Lisa Olsson, Elin Pettersen Sørgjerd and Bahareh Rasouli. The HUNT study is a collaboration between HUNT Research Centre (Faculty of Medicine, Norwegian University of Science and Technology), Central Norway Health Authority, Nord-Trøndelag County Council and the Norwegian Institute of Public Health. GlaxoSmithKline Norway supported the Diabetes Study at HUNT2 and HUNT3 financially through the Norwegian University of Science and Technology.

\section{REFERENCES}

1. Tuomi T, Carlsson A, Li H, Isomaa B, Miettinen A, Nilsson A, Nissén M, Ehrnström BO, Forsén B, Snickars B, Lahti K, Forsblom C, Saloranta C, Taskinen MR, Groop LC. Clinical and genetic characteristics of type 2 diabetes with and without GAD antibodies. Diabetes 1999; 48: 150-7.

2. Groop L, Tuomi T, Rowley M, Zimmet P, Mackay IR. Latent autoimmune diabetes in adults (LADA) - more than a name. Diabetologia 2006; 49: 1996-8.

3. Chiu HK, Tsai EC, Juneja R, Stoever J, Brooks-Worrell B, Goel A, Palmer JP. Equivalent insulin resistance in latent autoimmune diabetes in adults (LADA) and type 2 diabetic patients. Diabetes Res Clin Pract 2007; 77 (2): 237-44.

4. Naik RG, Brooks-Worrell BM, Palmer JP. Latent autoimmune diabetes in adults. J Clin Endocrinol Metab 2009; 94: 4635-44.

5. Gale E. Latent autoimmune diabetes in adults: a guide for the perplexed. Diabetologia 2005; 48: 2195-9.

6. Sørgjerd EP, Midthjell K, Grill V. Anti-GAD positivity: its prevalence and clinical impact in a large nondiabetic population: results from the HUNT-study. Diabetologia 2012; 55 (Suppl 1): S41.

7. Sørgjerd EP, Skorpen F, Kvaløy K, Midthjell K, Grill V. Time dynamics of autoantibodies are coupled to phenotypes and add to the heterogeneity of autoimmune diabetes in adults: the HUNT study, Norway. Diabetologia 2012; 55: 1310-8.

8. Rolandsson O, Palmer JP. Latent autoimmune diabetes in adults (LADA) is dead: long live autoimmune diabetes! Diabetologia 2010; 53: 1250-3.

9. Pettersen E, Skorpen F, Kvaløy K, Midthjell K, Grill V. Genetic heterogeneity in latent autoimmune diabetes is linked to various degrees of autoimmune activity: results from the Nord-Trøndelag Health Study. Diabetes 2010; 59: 302-10.

10. Carlsson S, Midthjell K, Grill V. Influence of family history of diabetes on incidence and prevalence of latent autoimmune diabetes of the adult: results from the Nord-Trøndelag Health Study. Diabetes Care 2007; 30: 3040-5.

11. Carlsson S, Midthjell K, Tesfamarian MY, Grill V. Age, overweight and physical inactivity increases the risk of latent autoimmune diabetes in adults: results from the Nord-Trøndelag health study. Diabetologia 2007; 50: $55-8$.

12. Olsson L. Ahlbom A, Grill V, Midthjell K, Carlsson S. Sleep disturbances and low psychological well-being are associated with an increased risk of autoimmune diabetes in adults. Results from the Nord-Trøndelag Health Study. Diabetes Res Clin Pract 2012; 98: 302-11.

13. Olsson L, Ahlbom A, Grill V, Midthjell K, Carlsson S. High levels of education are associated with an increased risk of latent autoimmune diabetes in adults: results from the Nord-Trøndelag health study. Diabetes Care 2011; 34: 102-7. 
14. Rasouli B, Andersson A, Ahlbom A, Grill V, Midthjell K, Carlsson S. Smoking is associated with reduced risk of autoimmune diabetes in adults contrasting with increased risk in overweight men with type 2 diabetes: a 22 year follow-up of the HUNT Study. Diabetes Care (In press).

15. Rasouli B, Andersson A, Ahlbom A, Grill V, Midthjell K, Olsson L, Carlsson S. Alcohol consumption reduces the risk of type 2 diabetes and autoimmune diabetes in adults. Diabetic Medicine 2013; 30: 56-64.

16. Krokstad S, Langhammer A, Hveem K, Holmen T, Midthjell K, Stene T, Bratberg G, Heggland J, Holmen J. Cohort Profile: The HUNT Study, Norway. Int J Epidemiol 2012; doi: 10.1093/ije/dys095.

17. Andersen MK, Lundgren V, Turunen JA, Forsblom C, Isomaa B, Groop PH, Groop L, Tuomi Latent autoimmune diabetes in adults differs genetically from classical type 1 diabetes diagnosed after the age of 35 years. Diabetes Care 2010; 33: 2062-4.

18. Cervin C, Lyssenko V, Bakhtadze E, Lindholm E, Nilsson P, Tuomi T, Cilio CM, Groop L. Genetic similarities between latent autoimmune diabetes in adults, type 1 diabetes, and type 2 diabetes. Diabetes 2008; 57: 1433-7.

19. Hayes C, Kriska A. Role of physical activity in diabetes management and prevention. J Am Diet Assoc 2008; 108 (4 Suppl 1): S19-23.

20. Joosten MM, Beulens JW, Kersten S, Hendriks HF. Moderate alcohol consumption increases insulin sensitivity and ADIPOQ expression in postmenopausal women: a randomised, crossover trial. Diabetologia 2008; 51: 1375-81.

21. Eckel RH, Kahn SE, Ferrannini E, Goldfine AB, Nathan DM, Schwartz MW, Smith RJ, Smith SR. Obesity and type 2 diabetes: what can be unified and what needs to be individualized? J Clin Endocrinol Metab 2011; 96: $1654-63$.

22. Agardh E, Allebeck P, Hallqvist J, Moradi T, Sidorchuk A. Type 2 diabetes incidence and socio-economic position: a systematic review and meta-analysis. Int J Epidemiol 2011; 40 (3): 804-18.

23. Holmqvist BM, Lofman O, Samuelsson U. A low incidence of type 1 diabetes between 1977 and 2001 in south-eastern Sweden in areas with high population density and which are more deprived. Diabet Med 2008; 25: 255-60.

24. McKinney PA, Okasha M, Parslow RC, Law GR, Gurney KA, Williams R, Bodansky HJ. Early social mixing and childhood type 1 diabetes mellitus: a case-control study in Yorkshire. UK Diabet Med 2000; 17: 236-42.

25. Radtke MA, Midthjell K, Nilsen TI, Grill V. Heterogeneity of patients with latent autoimmune diabetes in adults: linkage to autoimmunity is apparent only in those with perceived need for insulin treatment: results from the Nord-Trøndelag Health (HUNT) study. Diabetes Care 2009; 32: 245-50.

26. Maruyama T, Tanaka S, Shimada A, Funae O, Kasuga A, Kanatsuka A, Takei I, Yamada S, Harii N, Shimura $\mathrm{H}$, Kobayashi T. Insulin intervention in slowly progressive insulin-dependent (type 1) diabetes mellitus. $J$ Clin Endocrinol Metab 2008; 93: 2115-21.

27. Steffes MW, Sibley S, Jackson M, Thomas W. B-cell function and the development of diabetes-related complications in the Diabetes Control and Complications Trial. Diabetes Care 2003; 26: 832-836.

28. Brophy S, Davies H, Mannan S, Brunt H, Williams R. Interventions for latent autoimmune diabetes (LADA) in adults. Cochrane Database Syst Rev 2011 (9): CD006165.

29. Hawa MI, Thivolet C, Mauricio D, Alemanno I, Cipponeri E, Collier D, Hunter S, Buzzetti R, de Leiva A, Pozzilli P, Leslie RD, Action LADA Group. Metabolic syndrome and autoimmune diabetes: action LADA 3. Diabetes Care 2009; 32: 160-4.

30. Isomaa B, Almgren P, Henricsson M, Taskinen MR, Tuomi T, Groop L, Sarelin L. Chronic complications in patients with slowly progressing autoimmune type 1 diabetes (LADA). Diabetes Care 1999; 22: 1347-53.

31. Romkens TE, Kusters GC, Netea MG, Netten PM. Prevalence and clinical characteristics of insulin-treated, anti-GAD-positive, type 2 diabetic subjects in an outpatient clinical department of a Dutch teaching hospital. Neth J Med 2006; 64: 114-8.

32. Björklund A, Grill V, Carlsson S, Groop L. Diabetes mitt emellan typ 1 och type 2 diabetes. LADA - näst största diabetesgruppen där bästa behandling är osäker. Läkartidningen 2008; 105: 1568-70.

33. Juutilainen A, Lehto S, Ronnemaa T, Pyorala K, Laakso M. Similarity of the impact of type 1 and type 2 diabetes on cardiovascular mortality in middle-aged subjects. Diabetes Care 2008; 31: 714-9.

34. Myhill P, Davis WA, Bruce DG, Mackay IR, Zimmet P, Davis TM. Chronic complications and mortality in community-based patients with latent autoimmune diabetes in adults: the Fremantle Diabetes Study. Diabet Med 2008; 25 (10): 1245-50.

35. Olsson L, Grill V, Midthjell K, Ahlbom A, Andersson T, Carlsson S Increased mortality in autoimmune diabetes in adults. Diabetologia 2012; 55: (Suppl 1): S75. 